\title{
Análisis crítico de la literatura. ¿En pacientes adultos con cáncer diferenciado de tiroides y progresión en los últimos 14 meses, el tratamiento con sorafenib comparado con placebo mejora la supervivencia? Estudio DECISION
}

\section{Alejandro Román-González', Álvaro Sanabria}

${ }^{1}$ Medicina Interna Hospital Universitario San Vicente Fundación. Residente de Endocrinología. Grupo Endocrinología y Metabolismo. Universidad de Antioquia. Medellín, Colombia

${ }^{2}$ Cirujano de Cabeza y Cuello. Hospital Pablo Tobón Uribe. Profesor Departamento de Cirugía. Universidad de Antioquia. Medellín, Colombia

Correspondencia: Álvaro Sanabria, Departamento de Cirugía. Facultad de Medicina. Universidad de Antioquia

Email: alvarosanabria@gmail.com

\section{Resumen}

Introducción: El carcinoma de tiroides es el cáncer endocrino más frecuente. Las opciones terapéuticas cuando hay enfermedad progresiva, sintomática y resistente al yodo radioactivo son mínimas y poco efectivas. El sorafenib es una terapia aprobada para estos pacientes con base en los resultados del estudio DECISION. Se hace una revisión crítica acorde con los principios de medicina basada en la evidencia del estudio que llevó a la aprobación de este medicamento.

Título del estudio: Sorafenib in radioactive iodine-refractory, locally advanced or metastatic differentiated thyroid cancer: a randomised, double-blind, phase 3 trial. Lancet. 2014;384(9940):319-28(1)

Métodos: Ensayo clínico aleatorizado, financiado por Bayer HealthCare Pharmaceuticals y Onyx Pharmaceuticals, asignación oculta.

Pacientes y resultados: 419 pacientes mayores de 18 años con cáncer de tiroides localmente avanzado o cáncer de tiroides diferenciado metastásico refractario a yodo (papilar, folicular, incluyendo el de células de Hürthle y pobremente diferenciado), con progresión en los últimos 14 meses de acuerdo con los criterios de RECIST, al menos una lesión medible por TAC o por RM, ECOG 0-2; con función renal, medular y hepática normal y TSH menor de 0,5 mIU/L. El cáncer de tiroides refractario a yodo se definió como la presencia de al menos una lesión sin captación de yodo o pacientes con tumores yodo captantes y, bien sea, progresión luego de un tratamiento con yodo radioactivo en los últimos 16 meses o progresión luego de dos tratamientos con yodo recibidos con menos de 16 meses de diferencia (la última dosis recibida más de
16 meses antes) o una dosis acumulada de yodo de al menos 22,3 $\mathrm{GBq}$ ( $\geq 600 \mathrm{mCi}$ ). Se excluyeron pacientes que habían recibido previamente terapia dirigida, talidomida o quimioterapia para cáncer de tiroides, cirugía previa o trauma en los 30 días previos al inicio del medicamento, antecedente de cáncer de piel, cérvix o vejiga en los últimos cinco años, cáncer indiferenciado de tiroides, úlceras, infección activa o sangrado en los últimos tres meses, historia de hemorragia, infiltración traqueal, bronquial o esofágica; cardiopatía o hipertensión arterial no controlada, infección por VIH o hepatitis, embarazo o lactancia y alergia al sorafenib. Se permitieron dosis bajas de quimioterapia para radiosensibilización. El desenlace primario fue la supervivencia libre de progresión (cada ocho semanas) con criterios RECIST y los secundarios fueron supervivencia global, tiempo para la progresión, tasa de respuesta objetiva (parcial o completa), tasa de control de la enfermedad (parcial, completa o estable $\geq 4$ semanas/6 meses) y duración de la respuesta. La supervivencia libre de progresión fue mayor en el grupo de sorafenib que en el grupo de placebo (10,8 meses vs. 5,8 meses, HR 0,59 - IC 95\% 0,45-0,76 p<0,0001). El grupo de sorafenib tuvo una mayor tasa de eventos adversos (98,6 vs. 87,6\%).

Conclusión: Sorafenib mejora significativamente la supervivencia libre de progresión comparado con placebo en pacientes con cáncer de tiroides diferenciado progresivo refractario a yodo radioactivo. La diferencia es pequeña. Los eventos adversos son frecuentes. No se reporta calidad de vida o mejoría sintomática. Esta terapia debe ser usada por personas expertas y debe evaluarse individualmente cada caso, agotando las opciones disponibles para el paciente antes de someterlo a los riegos del tratamiento.

Palabras claves: inhibidores de tirosina cinasa, carcinoma de tiroides, sorafenib, medicina basada en la evidencia.

\section{Abstract}

Introduction: Thyroid carcinoma is the most frequent endocrine cancer. There are minimal therapeutic options once the disease is metastatic, progressive, symptomatic and radioactive iodine resistant. Sorafenib is a recent approved therapy for these patients based on the results of the DECISION trial. A critical review of this study is done according to the principles of evidence-based medicine. 
Title of the study: Sorafenib in radioactive iodine-refractory, locally advanced or metastatic differentiated thyroid cancer: a randomised, double-blind, phase 3 trial. Lancet. 2014;384(9940):319$28^{(1)}$.

Methods: Randomized clinical trial. Financial support was by Bayer HealthCare Pharmaceuticals and Onyx Pharmaceuticals. Blinded randomization.

Patients and results: 419 patients older tan 18 years with locally advanced thyroid carcinoma or well differentiate or poorly differentiate radioactive resistant metastatic thyroid carcinoma (papillary, follicular, Hurthle's cell carcinoma and poorly differentiate), with progression over previous 14 months according to RECIST, with at least on measurable target lesion by CT or MR imaging, ECOG 0-2; normal kidney, medullar and liver function and TSH less than $0.5 \mathrm{mIU} / \mathrm{L}$. Resistant radio-active iodine thyroid carcinoma was defined as the presence of at least 1 lesion without iodine uptake or patients with iodine uptake and progression over previous 16 months or progression after two cycles of radio-active iodine with less than 16 months of difference (lass cycle should be administered more 16 months before) or an total radioactive iodine of $\geq 600 \mathrm{mCi}$. Patients who were treated before with target therapy, talidomide or systemic chemotherapy were excluded. Also patients with surgery or trauma 30 days before starting sorafenib, personal history of skin, cervical or bladder cancer, undifferentiated thyroid carcinoma, ulcers, active infection or bleeding in previous three months, bleeding history, tracheal, bronchial or esophageal infiltration, cardiomyopathy, uncontrolled high blood pressure, HIV infection, hepatitis, pregnancy or breast feeding and sorafenib allergy were excluded. Small chemotherapy doses for radio sensitization were allowed. Primary outcome was progression free survival (every 8 weeks) according to RECIST and secondary outcomes was overall survival, time to progression, rate of objective response (partial or complete), rate of disease control (partial, complete or stable) and response duration. Progression free survival was larger in the sorafenib group than in the placebo group (10.8 month vs 5.8 months, HR 0.59 - IC 95\% 0.45-0.76 p<0.0001). Sorafenib group had a higher side effects frequency (98.6 vs 87.6\%).

Conclusion: Sorafenib significantly improves progression free survival compared with placebo in patients with radio-active iodine resistant progressive thyroid carcinoma. The difference is small. Side effects are frequent and quality of life and symptomatic improvement are not reported. This therapy must be used by experts and an individual assessment must be done and all available options must be used before consider the use of this treatment.

Key words: tyrosine kinase inhibitors, thyroid carcinoma, sorafenib, evidence-based medicine.

\section{Introducción}

El carcinoma papilar de tiroides es una epidemia en crecimiento $^{(2)}$. Para el año 2020 se considera que será el tercer cáncer en frecuencia en mujeres en edad fértil. Tan solo para este año se estima que, en Estados Unidos, se diagnosticarán 62.500 nuevos casos de carcinoma de tiroides ${ }^{(3)}$. A pesar de que la mayoría de los casos tienen un desenlace exitoso, con unas tasas elevadas de curación sólo con el manejo quirúrgico, un porcentaje de estos casos tendrá recurrencias loco-regionales y algunos de ellos tendrán enfermedad metastásica. Finalmente, el 5\% de los pacientes con carcinoma de tiroides morirán por esta causa ${ }^{(4)}$. El manejo de la enfermedad metastásica incluye el uso de terapias dirigidas, como el yodo radioactivo, el uso de radioterapia y el control regional con técnicas como la ablación con etanol o con radiofrecuencia. El uso de estas terapias está determinado por la tasa de progresión del tumor, la capacidad del tumor para captar yodo radioactivo, la disponibilidad de técnicas de radiología intervencionista, el tamaño y número de las metástasis, así como de los síntomas que presente el paciente. Un número mínimo del grupo general de pacientes con carcinoma bien diferenciado de tiroides va a tener enfermedad metastásica progresiva resistente a la terapia con yodo radioactivo. Este grupo de pacientes, a pesar de ser una minoría, no tiene opciones terapéuticas reales y el uso de quimioterapia citotóxica sistémica (doxorubicina, taxol y cisplatino) para el carcinoma diferenciado de tiroides sólo produce una respuesta parcial en el 25$38 \%$ de los casos, con remisiones completas muy infrecuentes ${ }^{(5-9)}$.

El uso de inhibidores de tirosina cinasa es una terapia dirigida y aprobada recientemente para el cáncer de tiroides diferenciado y pobremente diferenciado de tiroides, resistente a yodo radioactivo y en progresión ${ }^{(10)}$. La única terapia disponible y aprobada en nuestro medio es el sorafenib ${ }^{(11)}$. Durante la realización de este manuscrito fue aprobado por la FDA el lenvatinib ${ }^{(12)}$. En estudios fase II se están evaluando otras terapias moleculares ${ }^{(13,14)}$, como los inhibidores de BRAF (dabrafenib, vemurafenib) ${ }^{(15-17)}$ y los inhibidores de MEK como trametinib y selumetinib, entre muchos otros $^{(14,18-21)}$ (clinicaltriasl.gov).

Previo al inicio de cualquier terapia, el clínico debe hacer una lectura crítica de la evidencia presentada y definir si los estudios clínicos con los que se aprobó un tratamiento dado cumplen con los criterios más estrictos de la medicina basada en la evidencia y, según esto, definir la aplicabilidad del estudio en su práctica diaria. En este manuscrito se propone revisar desde la óptica de la medicina basada en la evidencia la investigación principal que llevó a la aprobación del sorafenib en cáncer de tiroides. Los criterios actuales para el uso de estas terapias deben incluir la no elegibilidad para manejo quirúrgico (finalmente una decisión de la capacidad del cirujano y del estadio funcional del paciente), la resistencia al yodo radioactivo, la progresión, el tamaño de la enfermedad y en particular la presencia de síntomas que afecten la calidad de vida del paciente ${ }^{(10)}$. En este sentido, debe tenerse en cuenta el principio primum non nocere, evaluando e involucrando en la decisión médica al paciente, explicándole los riesgos, a veces fatales, de esta terapia ${ }^{(10)}$. El uso inadecuado por personal no entrenado en el manejo de estas terapias puede provocar desenlaces peores que los que se buscan en el paciente.

\section{Síntesis de la evidencia}

Título del estudio: Sorafenib in radioactive iodine-refractory, locally advanced or metastatic differentiated thyroid cancer: a ran- 
domised, double-blind, phase 3 trial. Lancet. 2014;384(9940):319$28^{(1)}$.

Autores: Marcia Brose y colaboradores. Investigadores estudio DECISION. Ensayo multicéntrico internacional, 77 centros, 18 países.

Diseño: Ensayo clínico aleatorizado.

Fuentes de financiación: Bayer HealthCare Pharmaceuticals y Onyx Pharmaceuticals Asignación: Oculta.

Pacientes: 419 pacientes mayores de 18 años con cáncer de tiroides localmente avanzado o cáncer de tiroides diferenciado metastásico refractario a yodo (papilar, folicular, incluyendo el de células de Hürthle y pobremente diferenciado), con progresión en los últimos 14 meses acorde con los criterios de RECIST, al menos una lesión medible por TAC o por RM, ECOG 0-2; con función renal, medular y hepática normal y TSH menor de 0,5 mIU/L. El cáncer de tiroides refractario a yodo se definió como la presencia de al menos una lesión sin captación de yodo o pacientes con tumores yodo captantes y bien sea, progresión luego de un tratamiento con yodo radioactivo en los últimos 16 meses o progresión luego de dos tratamientos con yodo recibidos con menos de 16 meses de diferencia (la última dosis recibida más de 16 meses) o una dosis acumulada de yodo de al menos $22,3 \mathrm{GBq}$ ( $\geq 600 \mathrm{mCi}$ ). Se excluyeron pacientes que habían recibido previamente terapia dirigida, talidomida o quimioterapia para cáncer de tiroides, cirugía previa o trauma en los 30 días previos al inicio del medicamento, antecedente de cáncer de piel, cérvix o vejiga en los últimos cinco años, cáncer indiferenciado de tiroides, úlceras, infección activa o sangrado en los últimos tres meses, historia de hemorragia, infiltración traqueal, bronquial o esofágica; cardiopatía o hipertensión arterial no controlada, infección por VIH o hepatitis, embarazo o lactancia y alergia al sorafenib. Se permitieron dosis bajas de quimioterapia para radiosensibilización.

Intervención: Sorafenib $400 \mathrm{mg}$ cada 12 h (n=209) hasta progresión radiológica, toxicidad inaceptable o muerte, comparado con placebo $(n=210)$.

Desenlace primario: supervivencia libre de progresión (cada ocho semanas) con criterios RECIST: 1) Nuevas lesiones óseas; 2) $\geq 20 \%$ crecimiento y 3 ) Necesidad de radioterapia

Desenlaces secundarios: supervivencia global, tiempo para la progresión, tasa de respuesta objetiva (parcial o completa), tasa de control de la enfermedad (parcial, completa o estable $\geq 4$ semanas/6 meses) y duración de la respuesta.

\section{Resultados principales}

La supervivencia libre de progresión fue mayor en el grupo con sorafenib que en el grupo con placebo $(10,8$ meses vs. 5,8 meses, HR 0,59 - IC 95\% 0,45-0,76 p<0,0001). El grupo con sorafenib tuvo una mayor tasa de eventos adversos $(\mathbf{9 8 , 6}$ vs. $\mathbf{8 7 , 6 \% ) .}$

\section{Conclusión}

Sorafenib mejora significativamente la supervivencia libre de progresión, comparado con placebo en pacientes con cáncer de tiroides diferenciado progresivo refractario a yodo radioactivo.

\section{Análisis crítico del estudio}

¿Son los resultados válidos?

¿Los grupos de intervención y control iniciaron el estudio con el mismo pronóstico?

¿Se aleatorizaron los pacientes?

Sí. Se utilizó un sistema interactivo de voz y se estratificaron por edad (menores y mayores de 60) y región geográfica (Norteamérica vs. Europa vs. Asia).

¿Fue la aleatorización enmascarada?

Sí. Se utilizó un número único para cada paquete de tratamiento, el cual fue asignado por el sistema de voz interactivo. ¿En cada grupo, los pacientes fueron similares respecto a las variables pronósticas?

Sí, la tabla 1 muestra que no hubo diferencia estadísticamente significativa entre los grupos.

¿En cada grupo, los pacientes fueron similares respecto a las variables pronósticas?

¿Qué tipo de cegamiento se utilizó?

El estudio es doble ciego. La evaluación del desenlace de eficacia y los desenlaces secundarios fue realizada por un comité central independiente, utilizando los criterios RECIST.

¿Los grupos se mantenían balanceados respecto a sus factores pronósticos al terminar el estudio?

¿El seguimiento fue completo?

Sí. Sólo se identificaron cinco pérdidas y el desenlace principal se evaluó en el $100 \%$ de los pacientes.

¿Los pacientes se analizaron en el grupo en el que originalmente se aleatorizaron?

Los autores mencionan que se realizó un análisis por intención de tratamiento.

¿Se suspendió tempranamente el ensayo?

No.

¿Cuáles fueron los resultados?

¿Qué tan grande fue el efecto del tratamiento?

Pequeño. La mejoría en la supervivencia libre de progresión fue de cinco meses.

¿Qué tan preciso fue el estimativo del efecto del tratamiento?

Se encontró un hazard ratio (HR) de 0,59 (IC 95\%, 0,460,76; $\mathrm{P}<0,001$ ) para la supervivencia libre de progresión (SLP), con una prolongación en la mediana estadísticamente significativa (10,8 meses con Sorafenib (IC 95\% 9,1-12,9) vs. 5,8 meses con placebo (IC 95\% 5,3-7,8). La supervivencia global no fue diferente entre los grupos (HR 0,8, IC 95\% 0,54-1,19). La frecuencia de respuesta objetiva global (todas respuestas parciales) fue mayor en el grupo con sorafenib $(12,2 \%)$, en comparación con placebo $(0,5 \%)$. La frecuencia de control de la enfermedad (respuesta parcial más enfermedad estable) fue mayor en el grupo con sorafenib $(54,1 \%)$ que en el grupo con placebo $(33,8 \%)$.

¿Cómo puedo aplicar los resultados al cuidado de mis pacientes?

¿Fueron los pacientes del estudio similares a mis pacientes?

Los criterios de inclusión consideran pacientes que existen en la práctica habitual. 
¿Se consideraron todos los desenlaces importantes para el paciente? No. Los desenlaces relacionados con calidad de vida no fueron considerados. Tampoco se mencionan los aspectos de uso de recursos derivados del tratamiento.

¿Los beneficios esperados superan los riesgos y los costos?

No hay evaluación al respecto en el estudio. Los eventos adversos relacionados con la medicación fueron mayores que en el grupo placebo.

\section{Discusión}

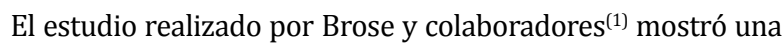
mejoría clínica en la supervivencia libre de progresión cuando se comparó sorafenib contra placebo en pacientes con cáncer diferenciado de tiroides metastásico y localmente avanzado en progresión, refractarios al yodo. La ganancia es pequeña (cinco meses), pero debe tenerse en cuenta, dada la ausencia de opciones reales para este grupo de pacientes ${ }^{(22)}$. Ahora bien, surgen varios interrogantes con el estudio y se presentan en tres secciones así: relacionadas con la población, relacionadas con el diseño y conducción del estudio y relacionadas con los resultados.

En relación con la población inicial incluida, la tabla 1 del estudio muestra que la gran mayoría tenían enfermedad metastásica (96\% para ambos grupos) y de éstos la mayoría de las metástasis ocurrieron en los pulmones. Pero llama la atención que cerca de la mitad tenían metástasis en los ganglios cervicales y hasta 15\% tenían metástasis en cabeza y cuello. Esto sugiere que hasta 15\% de los pacientes tenían en realidad una enfermedad locorregional avanzada y metástasis pulmonares. La pregunta es ¿por qué no se operaron estos pacientes en vez de llevarlos a terapia sistémica? Se acepta que, en estos casos, una cirugía con disminución del volumen tumoral cervical permite una mejor acción del yodo radioactivo sobre las metástasis sistémicas. Si a esto se le agrega que cerca del $40 \%$ de los pacientes habían recibido radioterapia, se fortalece la hipótesis de que un grupo importante de pacientes tenían enfermedad locorregional avanzada y que no fueron llevados a cirugía. Para los casos de tumores locamente avanzados, se ha demostrado que la resección local multiorgánica mejora ostensiblemente la supervivencia ${ }^{(23-26)}$. Esto indica que la población es bastante heterogénea y que asumir que el efecto del medicamento es igual para un paciente únicamente con enfermedad metastásica sistémica y control locorregional adecuado al de uno sin control locorregional, es difícil de admitir.

También llama la atención que un número relevante de pacientes $(11 \%$ en el grupo de sorafenib y $7 \%$ en el de placebo) tuvieron tumores pobremente diferenciados. Incluir este tipo de tumores, usualmente de comportamiento locorregional agresivo y combinarlos con pacientes con tumores papilares o foliculares metastásicos, dificulta aún más la extrapolación de las conclusiones. Todas estas suposiciones acerca de las diferentes subpoblaciones parecen verse confirmadas en la parte B de la figura 2 , donde los autores informan de algunos análisis de subgrupos.

También es interesante que se hayan combinado pacientes con menos de cinco lesiones y otros con más de 5 lesiones (confir- ma que hay subpoblaciones mezcladas) y con lesiones con tamaños tan disímiles (menores de $7 \mathrm{~cm}$ y mayores de $7 \mathrm{~cm}$ ).

Finalmente, en relación con la población seleccionada, el tiempo desde el diagnóstico, aunque igual entre los grupos (66 meses de mediana), es bastante amplio (de 4 meses a 400 meses). Esto refleja la inclusión de pacientes con enfermedad agresiva y rápidamente progresiva, cuyo pronóstico es diferente al de la subpoblación que ha sido tratada de un tumor tiroideo y que meses o años después presenta una recidiva regional o sistémica.

En relación con la conducción del estudio, la figura 1 permite observar varios aspectos que dejan interrogantes. El más importante es la cantidad de sujetos que suspendieron el tratamiento $(75 / 207,36 \%)$ en el grupo de sorafenib en comparación con el grupo de placebo $(22 / 209,10 \%)$, diferencia que es estadísticamente significativa. $40 \%$ de las suspensiones fueron por eventos adversos en el grupo de sorafenib (31/75) y 28\% por progresión de la enfermedad en vigencia de tratamiento (21/75) en comparación con $22 \%$ y $13 \%$ en el grupo de placebo. Incluso el número de muertes fue mayor en el grupo de sorafenib (12/207, 6\%), en comparación con placebo $(6 / 209,3 \%)$, aunque no estadísticamente significativo. Esto quiere decir que una vez iniciado el medicamento, la probabilidad de que el paciente obtenga un beneficio que sobrepase los riesgos es de sólo el $60 \%$, en razón de la aparición de eventos adversos o progresión. Además, la cantidad de pacientes que migraron del grupo placebo al de intervención es muy importante $(164 / 209,78 \%)$ y hace muy difícil interpretar los resultados relacionadas, con supervivencia global. Se sabe que estas migraciones masivas, donde la razón de la migración es indicada por el investigador, suelen estar sesgadas y tener efectos muy sugerentes cuando el desenlace que se escoge es la supervivencia libre de progresión ${ }^{(27-29)}$.

En relación con la razón riesgo-beneficio del medicamento pueden discutirse algunos puntos. Quizá el más relevante es la ausencia de datos acerca de la sintomatología y la calidad de vida de los pacientes al momento de iniciar el tratamiento. Cerca de $60 \%$ de los pacientes fueron clasificados como ECOG 0, lo que significa que eran poco sintomáticos y su enfermedad producía mínimo impacto en su estado funcional. Luego, la indicación del medicamento se enfocaba básicamente en evitar el crecimiento de las lesiones, en razón de que el desenlace primario correspondía a los criterios RECIST. Reconociendo la lentitud con que avanza la enfermedad tiroidea metastásica, es difícil justificar que se ofrezca un tratamiento con el fin de disminuir el diámetro de las lesiones, cuando se observa un número importante de eventos adversos y no se conoce el impacto que esta intervención pueda tener sobre un estado funcional adecuado ${ }^{(30-33)}$.

Aunque la tabla 2 muestra que el número global de eventos adversos es idéntico entre los grupos, la discriminación de cada uno de estos deja ver que el grupo de tratamiento tenía mayor número de eventos de síndrome mano-pie, diarrea, alopecia, exantema cutáneo, fatiga, pérdida de peso, hipertensión, anorexia, mucositis oral, prurito, náuseas y vómito, cefalea, constipación, neuropatía sensitiva, dolor abdominal, dolor de las extremidades y fiebre. En este sentido, no parece justificable el hecho de que 
un paciente asintomático y con buen estado funcional sea sometido a un tratamiento que produce alteraciones importantes en su condición sólo para evitar que las lesiones aumenten de tamaño (diferencia del 12\%). Incluso, la cantidad de pacientes que permanecen con enfermedad estable es similar entre los grupos (74\%), lo que hace pensar que el efecto del tratamiento ocurre incluso en un grupo menor de enfermos.

Las curvas de supervivencia que están llevadas a 800 días (un poco más de dos años), muestran que más del $80 \%$ progresó. Esto quiere decir que la mejoría en la supervivencia libre de progresión no alcanza para prolongar de manera importante la supervivencia en términos absolutos, aunque sí mantiene el efecto de los eventos adversos durante este tiempo.

En conclusión, el estudio ofrece una mejoría marginal en un desenlace no relevante para el paciente sin que se haya demostrado efecto en los desenlaces importantes para él cómo el estado funcional o la calidad de vida, pero con una probabilidad de presentar eventos adversos que pueden desmejorar su estado funcional, aun en casos en que este es adecuado. La heterogeneidad de las poblaciones hace difícil determinar cuáles son los mejores candidatos para usarlo, aunque parecen ser aquellos pacientes con enfermedad metastásica sistémica únicamente que sea resistente a yodo-radioactivo, que esté en progresión y donde la alteración del estado funcional y su potencial mejoría justifique los eventos adversos del tratamiento. La indicación para pacientes que presentan conjunción de enfermedad locorregional y sistémica no está muy clara. La decisión de uso del medicamento debe basarse en un análisis individual del paciente y bajo la evaluación de un equipo multidisciplinario, en el que se consideren la presencia de síntomas y el estado funcional del paciente y se tenga un entrenamiento adecuado en el manejo de este medicamento ${ }^{(10,34)}$.

\section{Referencias}

1. Brose MS, Nutting CM, Jarzab B, Elisei R, Siena S, Bastholt L, et al. Sorafenib in radioactive iodine-refractory, locally advanced or metastatic differentiated thyroid cancer: a randomised, double-blind, phase 3 trial. Lancet. 2014;384(9940):31928.

2. Tuttle RM, Haddad RI, Ball DW, Byrd D, Dickson P, Duh QY, et al. Thyroid carcinoma, version 2.2014. J Natl Compr Canc Netw. 2014;12(12):1671-80; quiz 80.

3. Siegel RL, Miller KD, Jemal A. Cancer statistics, 2015. CA Cancer J Clin. 2015;65(1):5-29.

4. Robbins J, Merino MJ, Boice JD, Ron E, Ain KB, Alexander HR, et al. Thyroid cancer: a lethal endocrine neoplasm. Ann Intern Med. 1991;115(2):133-47.

5. Droz JP, Schlumberger M, Rougier P, Ghosn M, Gardet P, Parmentier C. Chemotherapy in metastatic nonanaplastic thyroid cancer: experience at the Institut Gustave-Roussy. Tumori. 1990;76(5):480-3.

6. Ahuja S, Ernst H. Chemotherapy of thyroid carcinoma. J Endocrinol Invest. 1987;10(3):303-10.

7. Santini F, Bottici V, Elisei R, Montanelli L, Mazzeo S, Basolo F, et al. Cytotoxic effects of carboplatinum and epirubicin in the setting of an elevated serum thyrotropin for advanced poorly differentiated thyroid cancer. J Clin Endocrinol Metab. 2002;87(9):4160-5.

8. Gottlieb JA, Hill CS. Chemotherapy of thyroid cancer with adriamycin. Experience with 30 patients. N Engl J Med. 1974;290(4):193-7.

9. Gottlieb JA, Hill CS, Ibanez ML, Clark RL. Chemotherapy of thyroid cancer. An evaluation of experience with 37 patients. Cancer. 1972;30(3):848-53.

10. Carhill AA, Cabanillas ME, Jimenez C, Waguespack SG, Habra MA, Hu M, et al. The noninvestigational use of tyrosine kinase inhibitors in thyroid cancer: establishing a standard for patient safety and monitoring. J Clin Endocrinol Metab. 2013;98(1):31-42.

11. Brose MS, Nutting CM, Jarzab B, Elisei R, Siena S, Bastholt L, et al. Sorafenib in radioactive iodine-refractory, locally advanced or metastatic differentiated thyroid cancer: a randomised, double-blind, phase 3 trial. Lancet. 2014;384(9940):31928.

12. Schlumberger M, Tahara M, Wirth LJ, Robinson B, Brose MS, Elisei R, et al. Lenvatinib versus placebo in radioiodine-refractory thyroid cancer. $\mathrm{N}$ Engl J Med. 2015;372(7):621-30.

13. Cabanillas ME, Brose MS, Holland J, Ferguson KC, Sherman SI. A phase I study of cabozantinib (XL184) in patients with differentiated thyroid cancer. Thyroid. 2014;24(10):1508-14.

14. Dadu R, Devine C, Hernandez M, Waguespack SG, Busaidy NL, Hu MI, et al. Role of salvage targeted therapy in differentiated thyroid cancer patients who failed first-line sorafenib. J Clin Endocrinol Metab. 2014;99(6):2086-94

15. Dadu R, Shah K, Busaidy NL, Waguespack SG, Habra MA, Ying AK, et al. Efficacy and tolerability of vemurafenib in patients with BRAF(V600E) -positive papillary thyroid cancer: M.D. Anderson Cancer Center off label experience. J Clin Endocrinol Metab. 2015;100(1):E77-81.

16. Kim KB, Cabanillas ME, Lazar AJ, Williams MD, Sanders DL, Ilagan JL, et al. Clinical responses to vemurafenib in patients with metastatic papillary thyroid cancer harboring BRAF(V600E) mutation. Thyroid. 2013;23(10):1277-83.

17. Cabanillas ME, Patel A, Danysh BP, Dadu R, Kopetz S, Falchook G. BRAF Inhibitors: Experience in Thyroid Cancer and General Review of Toxicity. Horm Cancer. 2015;6(1):21-36.
18. Ho AL, Grewal RK, Leboeuf R, Sherman EJ, Pfister DG, Deandreis D, et al. Selumetinib-enhanced radioiodine uptake in advanced thyroid cancer. $\mathrm{N}$ Engl J Med. 2013;368(7):623-32

19. Hong DS, Cabanillas ME, Wheler J, Naing A, Tsimberidou AM, Ye L, et al. Inhibition of the Ras/Raf/MEK/ERK and RET kinase pathways with the combination of the multikinase inhibitor sorafenib and the farnesyltransferase inhibitor tipifarnib in medullary and differentiated thyroid malignancies. J Clin Endocrinol Metab. 2011;96(4):997-1005.

20. Bass MB, Sherman SI, Schlumberger MJ, Davis MT, Kivman L, Khoo HM, et al. Biomarkers as predictors of response to treatment with motesanib in patients with progressive advanced thyroid cancer. J Clin Endocrinol Metab. 2010;95(11):5018-27.

21. Lorch JH, Busaidy N, Ruan DT, Janne PA, Limaye SA, Wirth LJ, et al. A phase Il study of everolimus in patients with aggressive RAI refractory (RAIR) thyroid cancer (TC). J Clin Oncol. 2013;31(S1):abstr 6023.

22. Pitoia F. Sorafenib: is a partial response and stabilization of disease greater than $70 \%$ a modest response? Endocr Relat Cancer. 2014.

23. Gillenwater AM, Goepfert H. Surgical management of laryngotracheal and esophageal involvement by locally advanced thyroid cancer. Seminars in surgical oncology. 1999;16(1):19-29.

24. Kasperbauer JL. Locally advanced thyroid carcinoma. The Annals of otology, rhinology, and laryngology. 2004;113(9):749-53.

25. Mebed AH. Aggressive surgical therapy for locally invasive differentiated thyroid carcinoma : an experience of nineteen (19) cases. Journal of the Egyptian National Cancer Institute. 2007;19(4):282-91.

26. Mattavelli F, Bombardieri E, Collini P, Costa L, Pizzi N, Fallahadar D, et al. Role of surgery in treatment of advanced differentiated thyroid carcinomas. Acta otorhinolaryngologica Italica : organo ufficiale della Societa italiana di otorinolaringologia e chirurgia cervico-facciale. 2007;27(2):62-7.

27. Carroll KJ. Analysis of progression-free survival in oncology trials: some common statistical issues. Pharmaceutical statistics. 2007;6(2):99-113.

28. Panageas KS, Ben-Porat L, Dickler MN, Chapman PB, Schrag D. When you look matters: the effect of assessment schedule on progression-free survival. Journal of the National Cancer Institute. 2007;99(6):428-32.

29. Fleming TR, Rothmann MD, Lu HL. Issues in using progression-free survival when evaluating oncology products. Journal of clinical oncology : official journal of the American Society of Clinical Oncology. 2009;27(17):2874-80.

30. Fallowfield LJ, Fleissig A. The value of progression-free survival to patients with advanced-stage cancer. Nature reviews Clinical oncology. 2012;9(1):41-7.

31. Booth CM, Eisenhauer EA. Progression-free survival: meaningful or simply measurable? Journal of clinical oncology : official journal of the American Society of Clinical Oncology. 2012;30(10):1030-3.

32. Gutman SI, Piper M, Grant MD, Basch E, Oliansky DM, Aronson N. AHRQ Methods for Effective Health Care. Progression-Free Survival: What Does It Mean for Psychological Well-Being or Quality of Life? Rockville (MD): Agency for Healthcare Research and Quality (US); 2013.

33. Gemici C. Progression-free survival benefit or health-related quality-of-life advantage: which one to choose? Journal of clinical oncology: official journal of the American Society of Clinical Oncology. 2013;31(20):2635-6.

34. Brose MS, Frenette CT, Keefe SM, Stein SM. Management of sorafenib-related adverse events: a clinician's perspective. Semin Oncol. 2014;41 Suppl 2:S1-S16. 